\title{
Cell Proliferation Tracking Using Graphene Sensor Arrays
}

\author{
Ronan Daly, ${ }^{1}$ Shishir Kumar, ${ }^{1}$ Gyongyi Lukacs, ${ }^{2}$ Kangho Lee, ${ }^{1}$ Anne Weidlich, ${ }^{1}$ \\ Martin Hegner, ${ }^{2}$ and Georg S. Duesberg ${ }^{1}$ \\ ${ }^{1}$ CRANN and School of Chemistry, Trinity College Dublin, Dublin 2, Ireland \\ ${ }^{2}$ CRANN and School of Physics, Trinity College Dublin, Dublin 2, Ireland
}

Correspondence should be addressed to Ronan Daly, dalyr1@tcd.ie and

Martin Hegner, martin.hegner@tcd.ie

Received 15 June 2011; Revised 25 August 2011; Accepted 25 August 2011

Academic Editor: Maria Tenje

Copyright (C) 2012 Ronan Daly et al. This is an open access article distributed under the Creative Commons Attribution License, which permits unrestricted use, distribution, and reproduction in any medium, provided the original work is properly cited.

\begin{abstract}
The development of a novel label-free graphene sensor array is presented. Detection is based on modification of graphene FET devices and specifically monitoring the change in composition of the nutritive components in culturing medium. Microdispensing of Escherichia coli in medium shows feasibility of accurate positioning over each sensor while still allowing cell proliferation. Graphene FET device fabrication, sample dosing, and initial electrical characterisation have been completed and show a promising approach to reducing the sample size and lead time for diagnostic and drug development protocols through a label-free and reusable sensor array fabricated with standard and scalable microfabrication technologies.
\end{abstract}

\section{Introduction}

Controlled monitoring of bacterial growth has long been essential both as a diagnostic tool and as a standard drug development testing procedure. Common laboratory techniques involve bacterial proliferation on a Petri dish or in solution, providing an excess supply of nutrition and a controlled environment while sampling regularly for parallel tests by optical techniques. The need for higher throughput testing, more rapid diagnoses, and a more efficient use of samples has led to the implementation of miniaturised well-plate techniques. However, the drive for continuous improvement along with the concurrent growth in nanotechnology has led to a paradigm shift in sensing of biological activity. Significant advances in the coupling of proliferation to microcantilever [1-3] or quartz crystal microbalance (QCM) $[4,5]$ measurements have shown the potential for sensitivity to ultrasmall quantities of cells. In this work we are focused on the incorporation of graphene into label-free field effect transistor (FET) sensors to offer an alternative path to monitoring cell growth. In the approaches mentioned, it is the bacterium, the least abundant component, that acts as the analyte. Here we present initial results for the development of a novel label-free sensor for biological activity and specifically cell proliferation that relies upon measuring the change in the components of the bulk nutritive liquid. We propose the use of a scalable graphene FET microfabrication technology to (i) grow graphene films by chemical vapour deposition, (ii) transfer them to functional substrates and (iii) microstructure and contact graphene devices. These graphene FETs are functionalised by direct microdispensing of biological materials. We show initial evidence for cell proliferation on the microfabricated devices and the change in graphene charge transport responses with concentration changes of the lysogeny broth (LB) medium. This provides the basis for a scalable system allowing in situ tracking over the culture lifecycles in a range of parallel devices without the need for repeated sampling.

For diagnostics and drug development, one of the key drivers in sensor development is the reduction of the required sample volume. In a similar way that Moore's law drives the trend in decreasing transistor size for optimised device speed, there is a consistent decrease in sensor dimensions used for detecting proliferating bacteria. It has been noted in the literature that with a decrease in sample volumes there is an expected decrease in testing time. This is due to a number of factors, including the more rapid diffusion of nutrients because of the exponentially smaller system dimensions and the increased sensitivity requiring fewer lifecycles before detection occurs. This has been exploited 


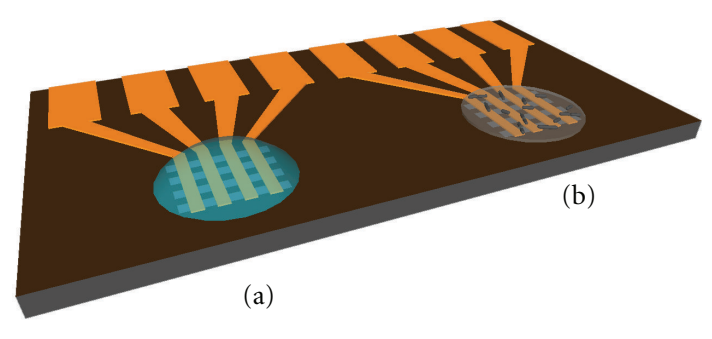

Figure 1: Metal electrodes contacting an underlying graphene layer acting as a sensor device with (a) bacteria proliferation occurring within a dosed volume of LB medium and (b) bacteria proliferation occurring on a thin agar layer, filled with a nutritive medium.

previously using micromechanical approaches, for example, by Gfeller et al. [1] where bacteria grew on an agar layer of a microcantilever array. However, these methods still have some drawbacks, namely, multistep fabrication techniques with limited device reusability and intricate surrounding measurement apparatus. For the devices proposed in this work, as shown in Figure 1, the sensor area is in complete contact with the sample and, through rapid diffusion and convection in such small sample volumes, is expected to be highly sensitive to changes. Large arrays of these sensors enable multiple parallel testing and improvement of the statistical confidence while still decreasing the batch time and conserving the low sample volume requirements. The robust nature of the graphene FETs allows repeated cleaning and reuse while the output is a simple electrical resistance measurement in the $\mathrm{k} \Omega$ range. In standard laboratory techniques and the microfabricated devices shown in Figure 1, $\mathrm{LB}$ is used as a feedstock to promote the binary fission process and bacterial growth on an agar layer or in solution. The nutrition contained within the aqueous broth includes vitamins, minerals, and organic compounds such as amino acids all of which are essential to the proliferation and growth of Escherichia coli (E. coli), the bacteria examined in this report.

The growth of cells is most often monitored by optical density (OD) measurements, where light absorption is used to identify the presence of bacteria in suspension. There are bulk-scale techniques to monitor the change in the LB content as a means to understanding the growth rate of bacteria. These track solution conductivity, $\mathrm{pH}$, or fluorescence [6], but there are to-date limited attempts to scale this approach down to microscale arrays and to our knowledge no attempts to incorporate two-dimensional carbon sensors for this purpose. The unique electrical and mechanical properties of graphene lend themselves to incorporation into FET devices in this case. The relative freedom from catalytic impurities, the low levels of noise, flexibility, robustness, and ease of microstructuring have all been noted [7] as benefits to using this material as an ultrasensitive recognition element in biosensor devices. Such devices have proven effective in air and liquids for the sensing of individual gas molecules [8], proteins [9], and bacteria [10] when direct graphene-analyte interactions occur. The direct contact is believed to lead to charge transfer and hence a change in the electrical response of the graphene sheet.

\section{Experimental Details}

2.1. Sensor Fabrication. Sensor devices are manufactured on $15 \times 15 \mathrm{~mm}$ pieces of p-doped (Boron) silicon (100) with a $300 \mathrm{~nm}$ layer of $\mathrm{SiO}_{2}$ from Si-Mat Silicon Materials, Germany, and cut using the Disco DAD 3220 wafer dicer. Samples are cleaned prior to microfabrication using ultrasonication in HPLC grade acetone, ultrasonication and rinse in HPLC grade propan-2-ol and subsequent drying in a rapid flow of filtered, dry nitrogen. An oxygen plasma treatment is also carried out to remove organic contamination using the Diener PICO barrel asher. Masks for UV lithography were designed in-house and created using the Heidelberg DWL 66FS direct writing system. UV lithography was carried out with the OAI Mask Aligner using Microposit S1813 positive photo resist and MF319 developer (both from Rohm and Haas Electronic Materials). Metal sputter deposition was carried out using the Gatan 682 Precision Etching Coating System at a rate of $0.1 \AA \mathrm{s}^{-1}$. After standard polymer lift-off procedures, residual polymer was removed by oxygen plasma treatment except when graphene was present, when solvent cleaning alone was used.

2.2. Graphene Transfer and Etching. Graphene, produced by chemical vapour deposition (CVD) as described in Results, is transferred from metal foil to the substrate as follows. A layer of poly(methyl methacrylate) (PMMA), (Mr-I 35 K PMMA from Microresist Technology $\mathrm{GmBH}$ ) was spin coated on top of graphene film/copper foil pieces. Thermal-release tape was adhered on top of this PMMA support film, and the copper was then etched by floating the sample in etchant $\left(0.25 \mathrm{M} \mathrm{FeCl}_{3}+0.2 \mathrm{M} \mathrm{HCl}\right)$. The resulting layered film of thermal-release tape/PMMA/graphene was cleaned with DI water, dried, and placed onto the substrate (as shown in Figure 3(b)). Because the graphene follows the contours of the PMMA/thermal-release tape layer, a uniform pressure was applied to the film to ensure close contact and conformation to the substrate. A range of pressures were used successfully ranging from 10 to 25 bar approximately. Heating the substrate from below promoted release of the upper tape layer. The remaining PMMA layer was removed by an initial soak in HPLC-grade acetone followed by an overnight soak in HPLC grade chloroform. The process can be carried out without thermal-release tape to avoid some contamination. In this case, PMMA-supported graphene is dredged from DI water onto the substrate. After the sample dries, PMMA can be removed as before.

2.3. Chemicals and Bacterial Culture. Chemicals and culturing medium were purchased from Sigma Aldrich (Arklow, Ireland) unless otherwise stated. E. coli CIP 53.126 was obtained from Collection de l'Institut Pasteur (Paris, France). Overnight cultures were prepared $\left(200 \mathrm{rpm}, 35^{\circ} \mathrm{C}\right.$, $15-18 \mathrm{~h})$ in $\mathrm{LB}(1 \% \mathrm{NaCl}, 1 \%$ tryptone, $0.5 \%$ yeast extract) from single colonies of E. coli. $1 \mathrm{~mL}$ of the overnight cultures were transferred into $30 \mathrm{~mL}$ of $50 \% \mathrm{LB}$, and $25 \%$ glycerol, $25 \%$ DI water and cultured $\left(200 \mathrm{rpm}, 35^{\circ} \mathrm{C}\right)$ for $110 \mathrm{~min}$ in order to reach a logarithmic growth rate. Glycerol was added 


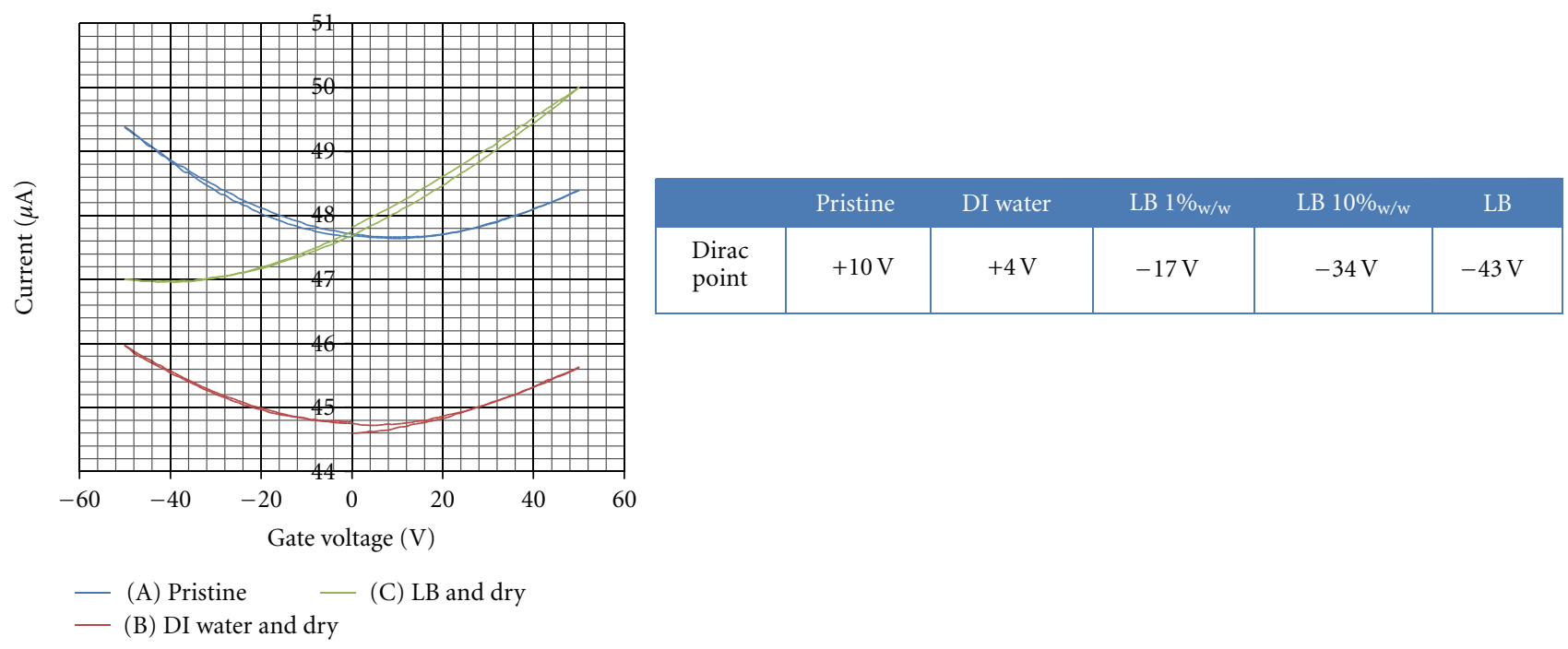

FIGURE 2: Performing gate sweep using back-gated graphene FET device shows that with exposure to LB medium there is a shift in the Dirac curve towards the negative bias voltage.

to ensure droplets did not evaporate prior to measurement. Observations confirmed evaporation was inhibited. Two additional diluted solutions were prepared from the LB stock. The first solution consisted of $500 \mu \mathrm{L} \mathrm{LB}, 250 \mu \mathrm{L}$ glycerol, and $250 \mu \mathrm{L}$ DI water. The second, more dilute solution consisted of $31 \mu \mathrm{L} \mathrm{LB}, 250 \mu \mathrm{L}$ glycerol, and $469 \mu \mathrm{L}$ deionised water (twice dilution and thirty-two times dilution, resp.). These are referred to as $2 \mathrm{LB}$ and $32 \mathrm{LB}$ in the text.

2.4. Microdispensing and Electrical Measurement. LB medium both with and without cells was dosed during this work using an Autodrop microdispensing system from Microdrop Technologies and a nozzle with a diameter of $50 \mu \mathrm{m}$. Subsequent electrical measurements on the graphene FET devices were carried out using a Keithley 2400 Sourcemeter attached to a Karl Suss probe station. Substrates were transferred between the dosing and measurement devices within a Petri dish containing a pad saturated with water to maintain humidity and inhibit evaporation of the dosed droplets during transport.

2.5. Additional Analysis. Raman spectroscopy was carried out using a Horriba Jobin Yvon LabRam HR system and a line of $632.8 \mathrm{~nm}$. Scanning electron microscopy (SEM) was carried out using the Zeiss ULTRA Plus in the Advanced Microscopy Laboratory, CRANN, Trinity College Dublin. Prior to SEM imaging, bacteria were fixed by soaking in $5 \% \mathrm{v} / \mathrm{v}$ glutaraldehyde solution in $0.05 \mathrm{M}$ phosphate buffer $(\mathrm{pH} 7)$ and incubated at room conditions with gentle agitation for 3-4h. Glutaraldehyde was then removed by 6 successive washes in fresh $0.05 \mathrm{M}$ phosphate buffer, each of 10 minutes duration. Samples were subsequently dehydrated with a sequence of 10-minute rinses in 10,30,50,70, 90, 100, and $100 \% \mathrm{v} / \mathrm{v}$ ethanol.

\section{Results and Discussion}

The fundamental premise of LB components affecting the conductance of graphene was confirmed using high-quality graphene flakes grown on $\mathrm{Ni}$ by chemical vapour deposition (CVD) and contacted with $e$-beam lithography. The graphene preparation and contacting process is described elsewhere [11]. The crucial step in this case is that the graphene has been cleaved by the Scotch tape to leave a clean surface. By dosing (i) $18 \mathrm{M} \Omega$ deionised water and (ii) LB medium onto a graphene FET device and comparing the electrical response of the sensor upon solvent evaporation, we see the precipitated materials from the LB medium lead to a slight increase in the measured resistance of the graphene strips and a clear shift in the Dirac point, as indicated in Figure 2. Graphene has linear dispersion in both valence and conduction bands. The degenerate point where these bands meet is known as the Dirac point. The Fermi level of graphene can move across the Dirac point under a bias, changing the concentration of charge carriers and therefore the resistance of samples. Thus, the minimum conductance (or maximum resistance) point observed in I-V characteristics displayed in Figure 2 corresponds to the Dirac point. A negative shift, as is observed for the samples exposed to $L B$, is equivalent to $n$-doping of the graphene.

With the development of a bulk graphene manufacturing technique, namely CVD, the incorporation of this remarkable material into scalable production of devices is now feasible [12]. The graphene used in this sensing application was grown by CVD, and all patterning was carried out by another scalable production technique, that of optical lithography. The CVD growth was carried out on $15 \times 15 \mathrm{~mm}$ samples of copper foil in a tube furnace, as indicated in Figure 3(a) and described in detail in a separate publication [13]. For this work two techniques are detailed in the experimental section 


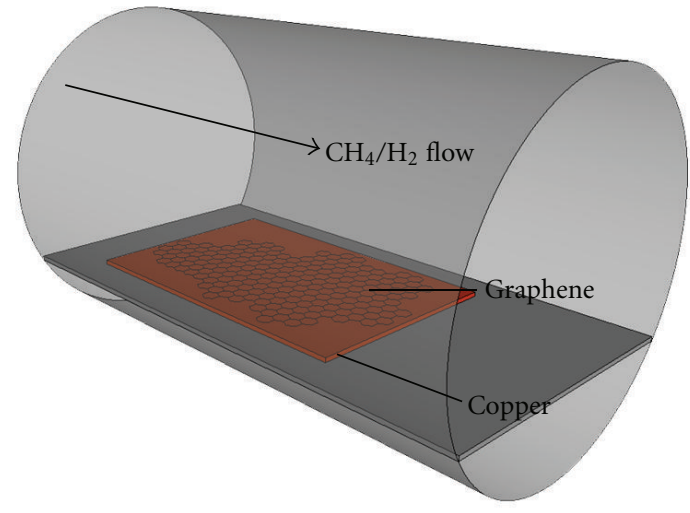

(a)

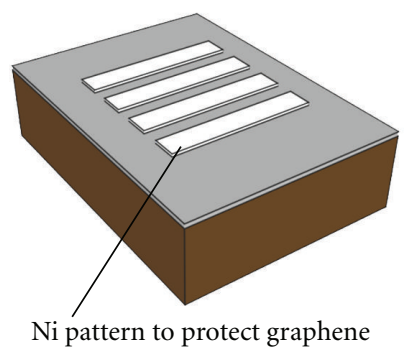

(c)

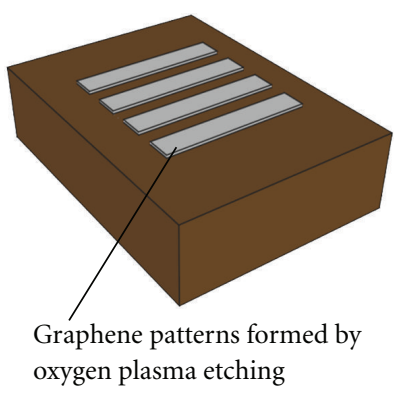

(d)

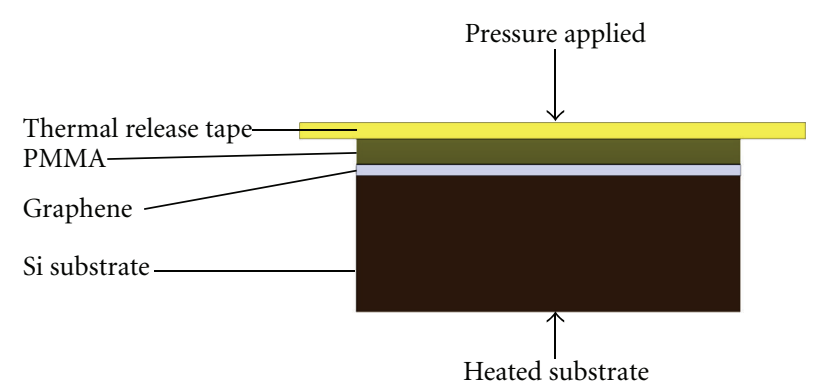

(b)

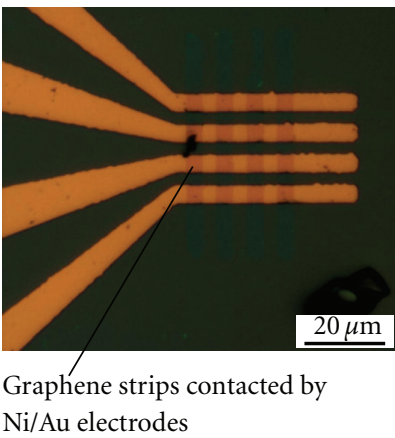

(e)

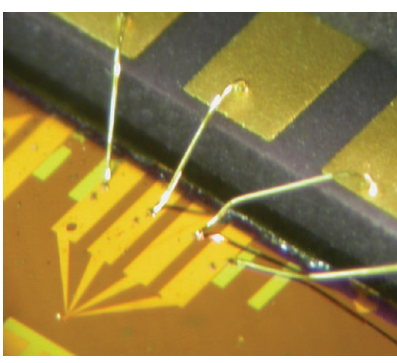

Devices can be wire-bonded to chip carrier for analysis

(f)

Figure 3: Production and microfabrication of graphene. (a) CVD growth of graphene on copper foil in a tube furnace, (b) graphene is attached to $\mathrm{SiO}_{2} / \mathrm{Si}$ by attaching to a support layer of PMMA and thermal-release tape and transferring by a combination of heat and pressure, (c) after transfer to $\mathrm{SiO}_{2} / \mathrm{Si}$ substrate, a Ni protection layer is patterned on the graphene, (d) oxygen plasma removes the unprotected graphene and the Nickel is subsequently removed by $\mathrm{HCl}$ etching. (e) shows the contacted graphene strips and (f) shows integration of the sensor into a chip carrier.

attempting to optimise the transfer of approximately $15 \times$ $15 \mathrm{~mm}$ films of graphene to the $\mathrm{SiO}_{2} / \mathrm{Si}$ substrates. In summary, after coating the graphene film/copper foil pieces with a PMMA support layer and a further layer of thermal release tape, the copper can be removed by etching with $\mathrm{FeCl}_{3}$. Transfer of graphene to $\mathrm{SiO}_{2} / \mathrm{Si}$ substrates is completed by applying pressure through the tape and PMMA support layers, pressing the graphene surface onto the substrate as indicated in Figure 3(b). Heat applied through the substrate allows easy release of the thermal-release tape leaving behind the PMMA/graphene layers with the graphene adhering to the substrate very strongly by the van der Waals forces [14]. The PMMA can be removed with solvent cleaning. Due to concerns regarding contamination from the thermal-release tape and the fracturing effects of the mechanical transfer method, a second approach was developed. The PMMA support layer is still applied to the graphene film/copper foil pieces and the etching occurs as before at the liquidair interface, leaving a graphene/PMMA layer floating on the surface. This is carefully transferred to the substrate surface through dip coating, and the same solvent cleaning steps occur to remove PMMA. The substrates have been prestructured by UV lithography with distinct, chromium alignment marks. These were included to enable a sequence of UV lithography patterning steps to occur that lead to metal-contacted graphene strips with good adhesion to the substrate, using a technique described by Kumar et al., [13]. As shown in Figures 3(c)-3(f), a sacrificial masking pattern of nickel is formed to protect the areas required for the devices and the uncovered graphene is removed by an oxygen plasma. The nickel protection layers are then completely removed by an acid etch with $1 \mathrm{M} \mathrm{HCl}$, and the remaining graphene strips are contacted by four $\mathrm{Ni} / \mathrm{Au}$ electrodes $(4 \mu \mathrm{m} / 48 \mu \mathrm{m})$ using a final UV lithography step. The contacted samples can then be probed directly using a needle prober or wire-bonded to a chip carrier for electrical measurements. This technique was modified from previous work to include a range of alignment marks for accurate positioning of all layers and a design that can be directly incorporated into an inkjet dosing system.

It is observed that the gate voltage behaviour and the scale of resistances recorded for graphene prepared with this technique have changed. This is partly due to the known issue of contamination during the incorporation of graphene into functional devices using multistep lithography processes. Graphene is notoriously difficult to maintain free of contamination and defects, and novel cleaning techniques will become essential for large-scale manufacture of graphene 


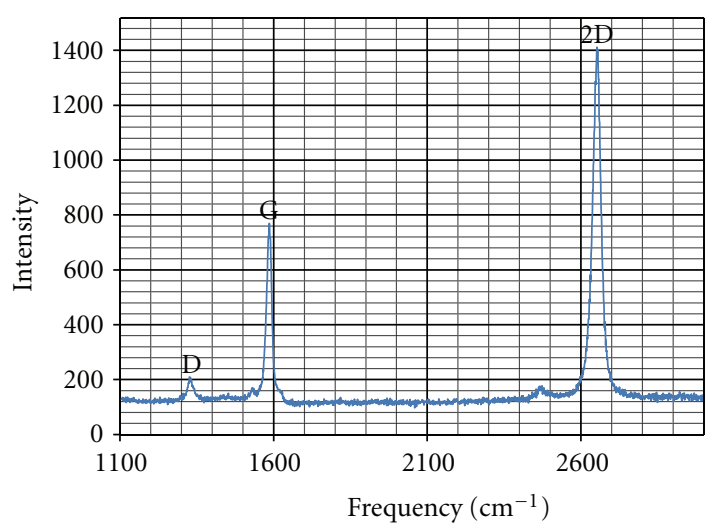

(a)

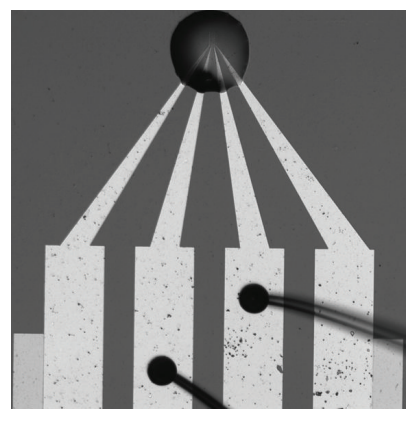

(b)
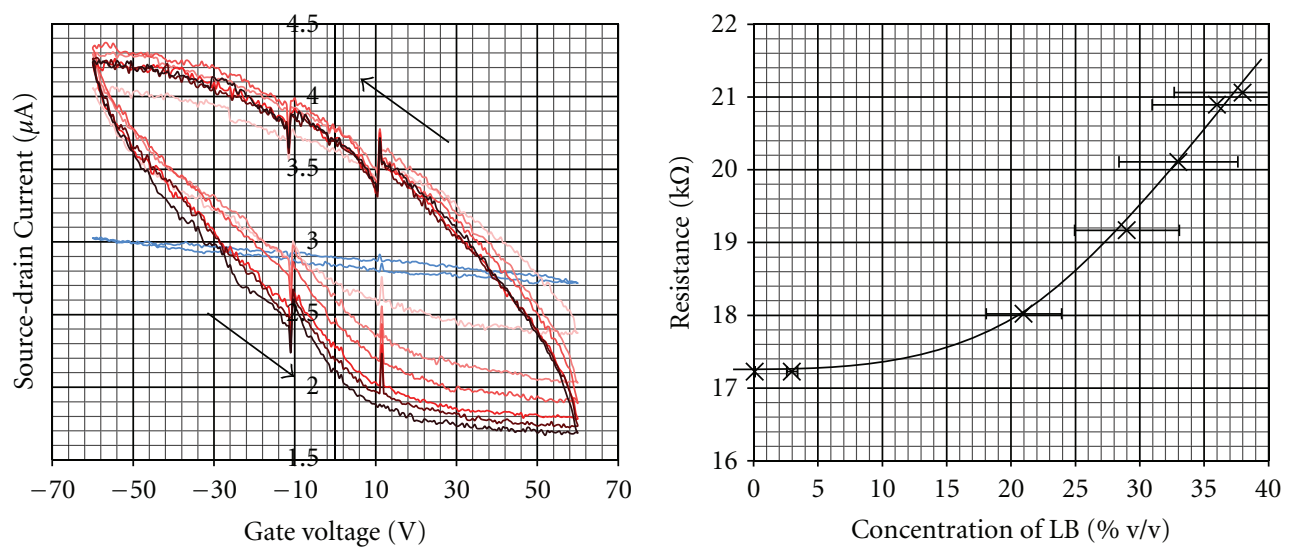

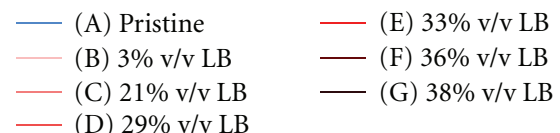

(c) (d)

Figure 4: (a) Example of the Raman spectrum of CVD-grown graphene after transfer. (b) Controlled deposition of small volumes of $\mathrm{LB} /$ glycerol medium is shown to be accurate by optical microscopy. (c) The p-type behaviour of the pristine graphene is observed, and a clear increase in hysteresis is noted upon measurement of LB. A shift in gate dependency with LB concentration is also noted. The glycerol is added to reduce droplet evaporation and is maintained at the same concentration in each case. (d) A change in resistance with LB concentration is noted using two-probe measurements on the graphene FET. Error bars are calculated based on droplet repeatability findings by Lukacs et al. [19].

[15]. An example of the Raman spectrum of CVD growth of graphene on copper that was transferred to $\mathrm{SiO}_{2}$ is shown in Figure 4(a). The G and 2D bands are clearly visible. The small bandwidth and the high $2 \mathrm{D} / \mathrm{G}$ ratio are indicative of single-layer graphene [16]. A small D-band is also observed around $1350 \mathrm{~cm}^{-1}$ indicating some defects/disorder present in our samples. Also, the unintentional doping of graphene due to the local environment or the substrate is a known phenomenon [17] and an observed $p$-type gate dependence of graphene is often attributed to this environmental factor [18]. This $p$-type behaviour is indicated in our results for pristine graphene shown in Figure 4(c), while no distinct Dirac point is found in the given gate voltage range. To understand how this modified graphene behaviour translates to a liquid sensing environment, a 32 times diluted LB medium (as described in Section 2) was dosed onto a sensor device using a microdispensing inkjet tool, as shown in Figure 4(b). To increase the LB concentration in this environment, additional drops were subsequently added containing a more concentrated solution (twice diluted LB). After each step change in concentration, the samples were transported to a probe station for electrical measurement as noted in Section 2.

A set of results using this method is presented in Figure 4(d). The increase in resistance with LB concentration is again noted. While intuitively, the inclusion of ions in solution would lead to a decrease in solution resistance, the observed increase in resistance with solution concentration is tentatively assigned to a charge transfer of negative charge from the LB solution to the graphene, counterbalancing its pristine $p$-type behaviour. This is consistent with the behaviour noted earlier for the dried LB scenario where there 


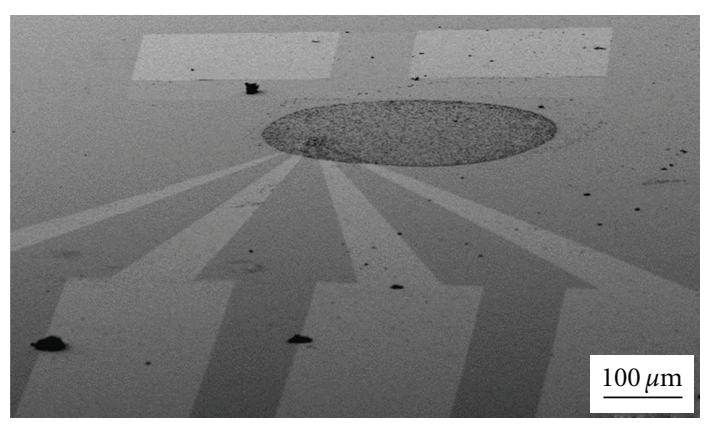

(a)

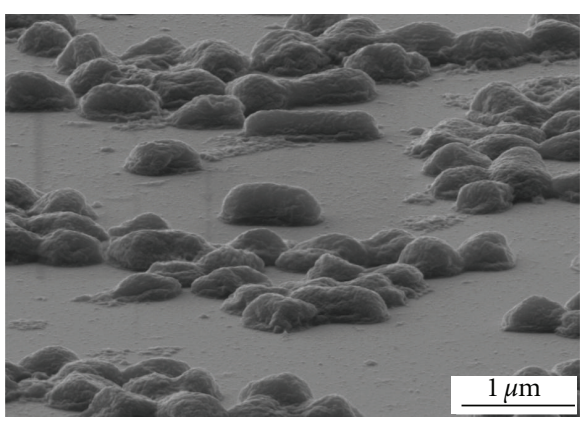

(b)

FIGURE 5: Microdispensing of 50\% LB/25\% glycerol medium containing E. coli shows it is feasible to guide proliferation to the graphene sensor region. (a) An SEM image shows the graphene sensor and contacts at $80^{\circ}$ tilt with a dense circular pattern of adhered bacteria fixed using a dehydration protocol and shown in (b) at a higher magnification.

is a shift in the Dirac point towards a negative gate voltage (Figure 2). As the result of this balance of charges, the system moves closer to neutrality and exhibits lower conductivity. Unlike single-molecule detection studies, the complexity of the medium does not allow a detailed interpretation. Each salt and biomolecule will contribute to the charge transfer in a different way, as will the balance struck between molecules converted to bacterial biomass and those excreted during proliferation. However, this initial approach allows insight into the potential sensitivity to changes in medium concentration.

With this proven ability to sense changes in a complex liquid system on top of graphene FET arrays, it was essential to show the capability to deliver in situ and localised bacteria proliferation. Through experiments microdispensing E. coli to sensor devices and comparing a device in an ambient atmosphere where the droplet is allowed to dry and a device maintained in an incubator for 1 day we show (i) survival of the E. coli through the dispensing protocol, (ii) no obvious ill effects of the substrate or possible contaminants from fabrication processes, and (iii) bacteria proliferation around the sensor area. Figure 5(a) shows an SEM image showing the dense drying pattern surrounding a graphene FET device made up of the E. coli shown in more detail by the second SEM image, see Figure 5(b). Imaging of the control and incubated samples shows an increase in cells/unit area by a factor of 8.6 over the course of 1 day. Fixation and drying steps, described in Methods, have been introduced to allow high-resolution images of cells in incubated samples.

\section{Future Work and Conclusions}

An optimised system for in situ electrical measurements is currently being developed. This has led to a system where both control and bioactive samples are dispensed within a single array and electrically analysed over the course of the experiment. Ongoing work is focusing on minimising background signals, optimising the device fabrication for high humidity environments and defining necessary sample concentrations to ensure a change within the detectable limits. As noted earlier, the rapid diffusion through such small volumes and the sensitivity of graphene-based sensors are expected to ensure short batch times. Future work will include cell growth on a nanoscale agar-coating layer directly over the graphene device. This will reduce the sample volume significantly and is expected to ensure a rapid measurement of bacteria proliferation. More fundamental work is required to isolate the influences of each component in the LB medium to quantify the contributing factors to the measurements as the nutrition is converted to biomass. During the initial rapid-growth stage of the bacterial proliferation process that we are targeting, the cells grow at the maximum rate for a given medium using the nutrition to form bacterial biomass. While in this initial work we assume this to be the main influence on changes to the solution properties, it is known that a range of metabolites are also produced and excreted in this stage. It is well understood from the fermentation industry that once the proliferation slows, the production of metabolites also changes leading to a variation in $\mathrm{pH}$ [20]. In fact the biomass can be estimated by the progressive change in $\mathrm{pH}$ this process causes [21]. Planned work also includes examination of additional influences from biomolecules excreted from the proliferating bacteria.

We have shown the feasibility of a label-free micronscale graphene sensor array, fabricated with standard and scalable technologies, for monitoring the change in concentration of the nutritive medium used to promote bacteria proliferation. The introduction of different concentrations of nutritive medium could immediately be analysed with this novel electrochemical sensing method, and clear shifts in conductivity were detected in the liquid environment. The device has been tested in liquid and a surrounding humid environment and shows minimal drift that can in future be accounted for with additional control sensors. It is believed that the ability to microfabricate the sensors towards the length scale of individual bacteria will in future allow the targeting of just a few of the organisms, thus providing very specific and quantitative data. We have developed a fabrication, microdispensing, and analysis protocol for this novel sensing approach and demonstrated that the indirect measurement technique will be suitable for inexpensive, reusable, and rapid diagnostic tools. 


\section{Acknowledgments}

This work was supported by the Science Foundation Ireland under the CSET scheme SFI08/CE/I1432. The SEM imaging was enabled by the Advanced Microscopy Laboratory, Trinity Technology and Enterprise Campus, Dublin 2, Ireland under the framework of the INSPIRE programme, funded by the Irish Government's Programme for Research in Third Level Institutions, Cycle 4, National Development Plan 2007-2013. GSD acknowledges SFI for the PICA grant and SK the Embark Initiative for an IRCSET scholarship.

\section{References}

[1] K. Y. Gfeller, N. Nugaeva, and M. Hegner, "Rapid biosensor for detection of antibiotic-selective growth of Escherichia coli," Applied and Environmental Microbiology, vol. 71, no. 5, pp. 2626-2631, 2005.

[2] D. Ramos, J. Tamayo, J. Mertens, M. Calleja, and A. Zaballos, "Origin of the response of nanomechanical resonators to bacteria adsorption," Journal of Applied Physics, vol. 100, no. 10, Article ID 106105, pp. 106105-1-106105-3, 2006.

[3] N. Nugaeva, K. Y. Gfeller, N. Backmann, H. P. Lang, H. J. Güntherodt, and M. Hegner, "An antibody-sensitized microfabricated cantilever for the growth detection of Aspergillus niger spores," Microscopy and Microanalysis, vol. 13, no. 1, pp. 13-17, 2007.

[4] N. Kim and I. S. Park, "Application of a flow-type antibody sensor to the detection of Escherichia coli in various foods," Biosensors and Bioelectronics, vol. 18, no. 9, pp. 1101-1107, 2003.

[5] X. L. Su and Y. Li, "A QCM immunosensor for Salmonella detection with simultaneous measurements of resonant frequency and motional resistance," Biosensors and Bioelectronics, vol. 21, no. 6, pp. 840-848, 2005.

[6] C. Faber, "Assessment of the inhibitory potency by MRI," in NMR Spectroscopy in Pharmaceutical Analysis, U. Holzgrabe et al., Ed., Elsevier, 2008.

[7] W. Yang, K. R. Ratinac, S. R. Ringer, P. Thordarson, J. J. Gooding, and F. Braet, "Carbon nanomaterials in biosensors: Should you use nanotubes or graphene," Angewandte Chemie_-International Edition, vol. 49, no. 12, pp. 2114-2138, 2010.

[8] F. Schedin, A. K. Geim, S. V. Morozov et al., "Detection of individual gas molecules adsorbed on graphene," Nature Materials, vol. 6, no. 9, pp. 652-655, 2007.

[9] Y. Ohno, K. Maehashi, Y. Yamashiro, and K. Matsumoto, "Electrolyte-gated graphene field-effect transistors for detecting ph and protein adsorption," Nano Letters, vol. 9, no. 9, pp. 3318-3322, 2009.

[10] N. Mohanty and V. Berry, "Graphene-based single-bacterium resolution biodevice and DNA transistor: Interfacing graphene derivatives with nanoscale and microscale biocomponents," Nano Letters, vol. 8, no. 12, pp. 44694476, 2008.

[11] P. N. Nirmalraj, T. Lutz, S. Kumar, G. S. Duesberg, and J. J. Boland, "Nanoscale mapping of electrical resistivity and connectivity in graphene strips and networks," NanoLetters, vol. 11, pp. 16-22, 2011.

[12] X. Li, W. Cai, J. An et al., "Large-area synthesis of high-quality and uniform graphene films on copper foils," Science, vol. 324, no. 5932, pp. 1312-1314, 2009.
[13] S. Kumar, N. Peltekis, K. Lee, H. Kim, and G. S. Duesberg, "Reliable processing of graphene using metal etchmasks," Nanoscale Research Letters, vol. 6, no. 1, p. 390, 2011.

[14] J. S. Bunch, S. S. Verbridge, J. S. Alden et al., "Impermeable atomic membranes from graphene sheets," Nano Letters, vol. 8, no. 8, pp. 2458-2462, 2008.

[15] N. Peltekis, S. Kumar, N. McEvoy, K. Lee, A. Weidlich, and G. S. Duesberg, "The effect of downstream plasma treatments on graphene surfaces," Carbon, vol. 50, no. 2, pp. 395-403, 2012.

[16] A. C. Ferrari, J. C. Meyer, V. Scardaci et al., "Raman spectrum of graphene and graphene layers," Physical Review Letters, vol. 97, no. 18, Article ID 187401, 2006.

[17] Y. H. Wu, T. Yu, and Z. X. Shen, "Two-dimensional carbon nanostructures: fundamental properties, synthesis, characterization, and potential applications," Journal of Applied Physics, vol. 108, no. 7, Article ID 071301, 2010.

[18] H. E. Romero, N. Shen, P. Joshi et al., "n-type behavior of graphene supported on $\mathrm{Si} / \mathrm{SiO} 2$ substrates," ACS Nano, vol. 2, no. 10, pp. 2037-2044, 2008.

[19] G. Lukacs, N. Maloney, and M. Hegner, "Ink-jet printing: perfect tool for cantilever array sensor preparation for microbial growth detection," Journal of Sensors. In press.

[20] M. Scheidle, B. Dittrich, J. Klinger, H. Ikeda, D. Klee, and J. Buchs, "Controlling $\mathrm{pH}$ in shake flasks using polymer-based controlled-release discs with pre-determined release kinetics," BMC Biotechnology, vol. 11, p. 25, 2011.

[21] M. L. Christensen and N. T. Eriksen, "Growth and proton exchange in recombinant Escherichia coli BL21," Enzyme and Microbial Technology, vol. 31, no. 4, pp. 566-574, 2002. 

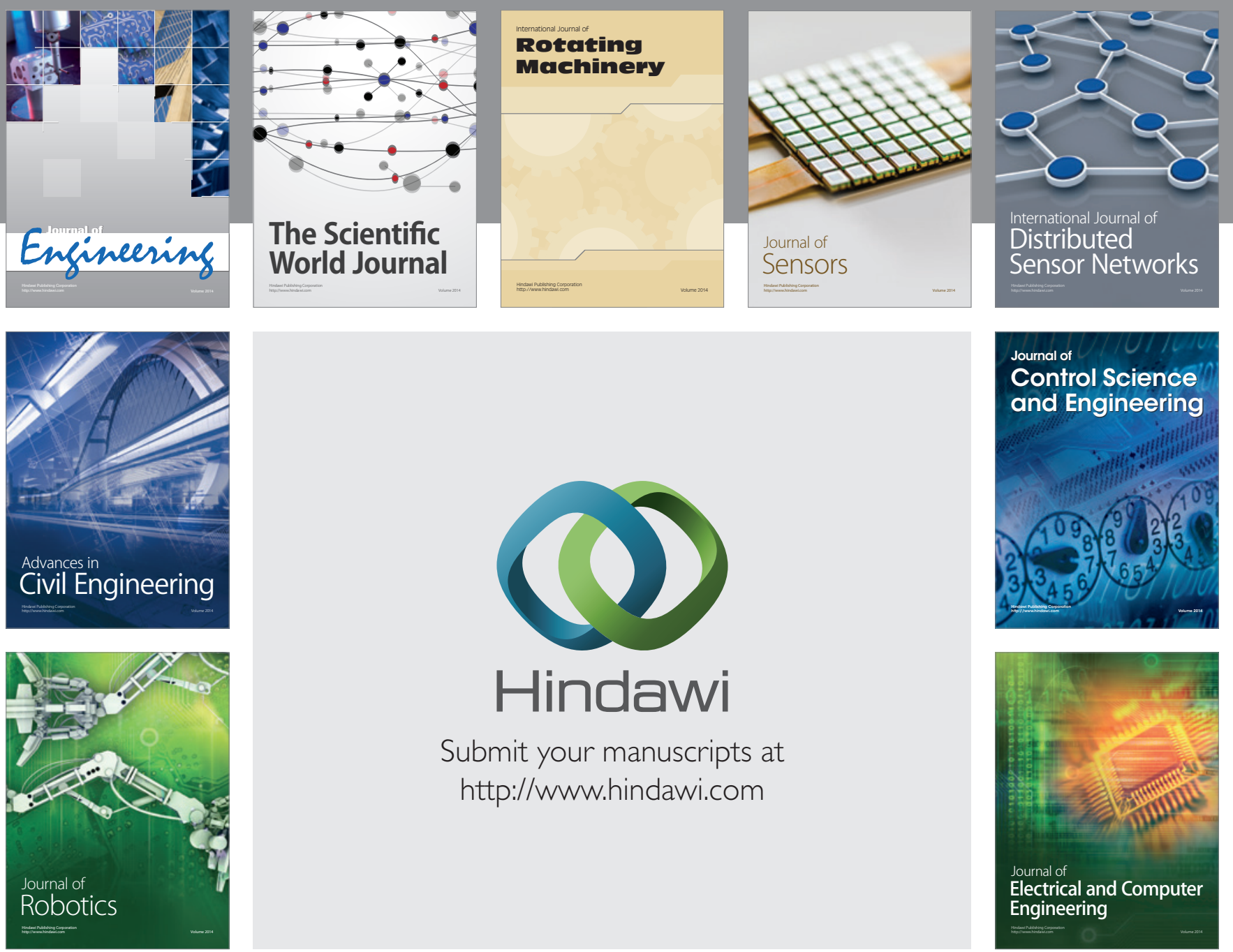

Submit your manuscripts at

http://www.hindawi.com
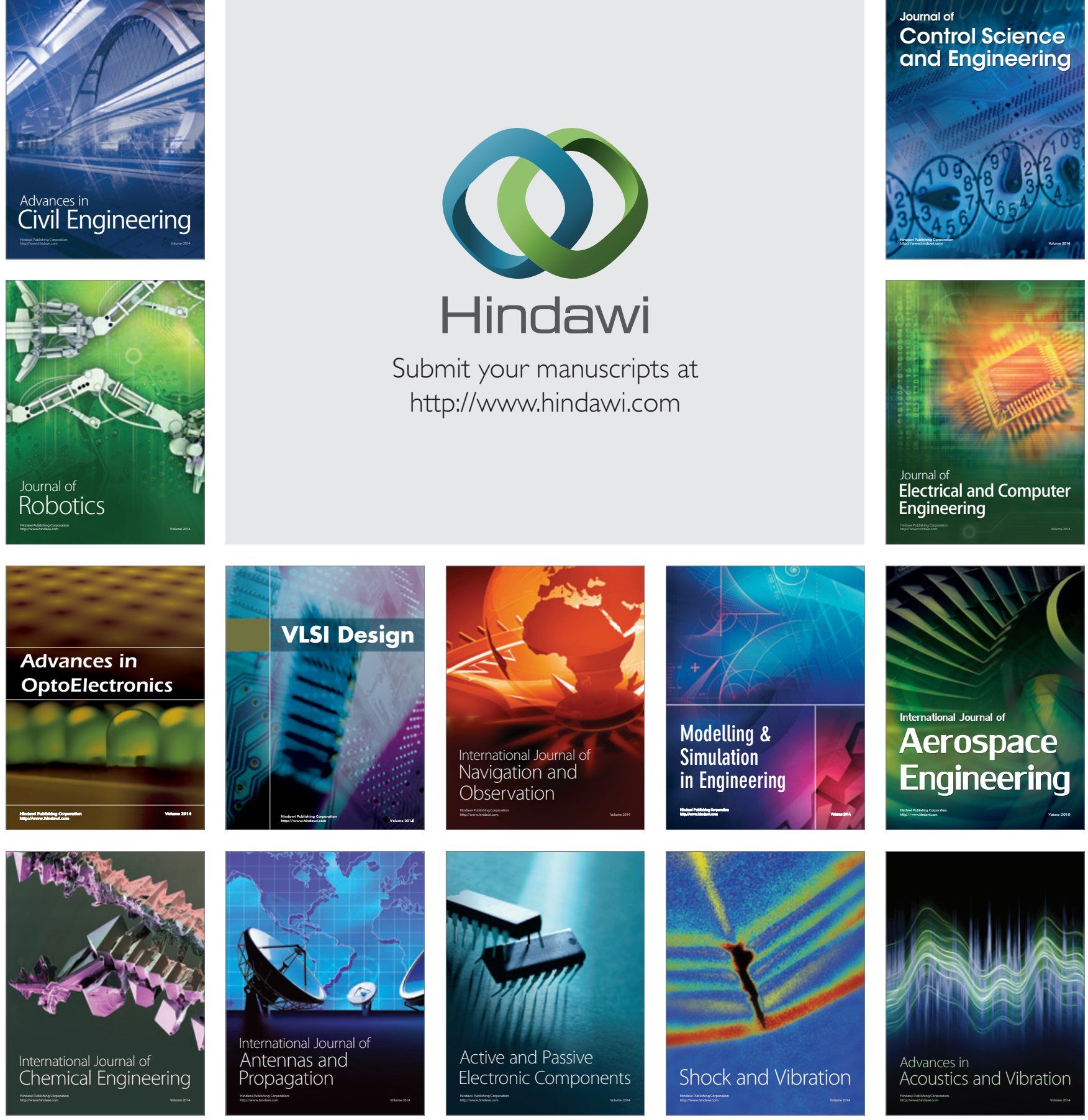